\title{
Д.К. Маслов
}

\section{ТРОП ОТНОСИТЕЛЬНОСТИ И «ДИАЛЕКТИЧЕСКАЯ СТРАТЕГИЯ» В ФИЛОСОФИИ СЕКСТА ЭМПИРИКА}

\begin{abstract}
Анализируется использование тропа относительности Секстом Эмпириком. Поднимается проблема возможного агностицизма главного представителя пирронизма и предлагаются аргументы и основания, которые показывают способ отведения данного возражения. Основой интерпретации является так называемая диалектическая стратегия, с помощцью которой Секст мог описать свое непропозициональное использование норм и аргументов, которые были им заимствованы у догматических философов и использованы против них самих.

Ключевые слова: троп относительности, диалектическая стратегия, античный пирронизм, Секст Эмпирик, самоопровержение.
\end{abstract}

В философских дискуссиях оппоненты регулярно стараются опровергать друг друга, указывая на противоречия в позиции или выведение неприемлемых следствий. Учение Секста Эмпирика не избежало подобного рода критики. В силу специфики философия пирронизма кажется уязвимой в нескольких ключевых пунктах, таких как вопрос о догме, возможности действовать и прочих [1. С. 139; 2, 3]. Аргументы, уличающие скептика-пиррониста в непоследовательности, противоречивости и самоопровержении, были хорошо известны еще в Античности, и Секст в своих трактатах приложил много усилий для их развенчания. Среди современных историков философии заметно преобладает тенденция разработки апологетических интерпретаций пирронизма. Опираясь на проблемные места, потенциально способные привести скептика к противоречию и самоопровержению, исследователи этого направления не обвиняют безоглядно пирронизм в самопротиворечии, но стараются реконструировать аргументы Секста в защиту пирронизма в соответствии с принципом доброжелательности [4]. Некоторые другие исследователи считают, что философия Секста Эмпирика, в сущности, была непоследовательна и самопротиворечива, что и делает ее непривлекательной, а аргументы ошибочными и самоопровергающими, а значит, недостойными внимания.

Рассмотрим один из ключевых моментов. Секст использует против своих оппонентов (по большей части против позитивных догматиков) ряд сильных аргументов, имеющих результатом доказательство невозможности познания вещей по природе. Это дает основание некоторым исследователям отстаивать взгляд, согласно которому Секст в действительности утверждал тезис о непознаваемости вещей (несмотря на его заявления о воздержании) и принадлежит, таким образом, к негативно догматическим философам ${ }^{1}$. Поскольку негативный догматизм явно несовместим с программой пирронизма, анти-

${ }^{1}$ Именно к такому выводу приходит J. Palmer [5]. G. Striker [6. Р. 58] также склоняется к признанию пирронизма негативным учением. Бёрниет считает, что скептик либо непоследователен, либо неискренен [7]. 
скептические аргументы, направленные на обоснование негативного характера скепсиса, представляют собой серьезную проблему для целостности пирронизма.

В общих чертах основу учения Секста Эмпирика можно описать таким образом. Целью скептика является достижение атараксии (ajtaraxi>a), душевного спокойствия и безмятежности (PH I 12, 25). Поэтому философия пирронизма занята постоянным воспроизведением однажды пройденного, последовательного пути ${ }^{1}$ к ней, который Секст Эмпирик описывает в начале «Пирроновых основоположений» ${ }^{2}$ (PH I 8-12, 26-27). Атараксия достигается с помощью исследования высказываний философов и ученых о неочевидных вещах, в результате чего скептик оказывается в ситуации равной убедительности спорящих друг с другом (т.е. противоречащих) аргументов по некоторому вопросу. Это приводит его к воздержанию от суждения, вслед за которым случайно (tucikw v) следует атараксия ${ }^{3}$.

Своих оппонентов-философов Секст называет догматиками и четко отделяет от них свою философию, выделяя среди догматиков негативных и позитивных. К первым относятся те, кто утверждает принципиальную непостижимость вещей по природе, к другим же причисляются те, кто располагает позитивной философской системой и утверждает, что отыскал истину вещей (PH I 1-3). Скептика же он определяет как «искателя» (PH I 7), который еще не нашел истину, как и доказательства невозможности ее познать, - скептик воздерживается от суждения, не имеет никаких догм (PH I 12-16), что косвенным путем обеспечивает ему душевное спокойствие. Отсюда утверждение или вывод, ведущие к тезису о непознаваемости вещей, явным образом бы противоречили философии пирронизма и делали бы недействительным способ достижения его цели, поэтому оно недопустимо внутри философии пирронизма. При этом важно, что скептик не исключает, что однажды истина будет найдена, как отмечает Секст (PH I 226). Но, может быть, Секст действительно маскирует свой агностицизм, который следует из его аргументации? ${ }^{4}$ Если Секст действительно в ходе рассуждения

${ }^{1}$ Гизела Страйкер использует для описания этого пути у Секста термин «история» (story) [8. P. 117-118].

2 Трактат «Пирроновы основоположения» обозначается для краткости как $P H$, а «Против ученых» как - $M$, где латинская цифра обозначает книгу, а арабская - строфу. Все ссылки на русский перевод даются по единственному русскому изданию [9], а цитаты на греческом по [10]. Ссылка на Диогена Лаэртского (далее - DL) дается по [11].

${ }^{3}$ Под случайностью здесь понимается ненамеренное следствие, которое нельзя достичь специальным усилием. Подчеркивается независимость наступления атараксии от эпистемических усилий философа. И если атараксия наступает после воздержания от суждения по исследуемому вопросу, именно к воздержанию будет стремиться скептик.

${ }^{4}$ Важно отметить то, что мы в данной работе опираемся исключительно на образ пирронизма, представленный Секстом в PH I. Американский исследователь Р. Бетт при анализе $M$ XI выявил там отличную от представленной позицию скептика, которую связал с влиянием Энесидема. [12]. В книге «Против этиков» Секст утверждает, что не существует добра и зла по природе, но лишь в отношении к определенной ситуации. Хотя Бетт и признает проблематичность, возникающую из сопоставления этих двух версий пирронизма у Секста, он предлагает рассматривать отрицание существования добра и зла по природе как определенный способ отказа от вынесения суждений о чем-либо как о добром или злом по природе. В такой интерпретации он видит иной возможный путь обоснования воздержания от суждения, нежели данный в PH I [Там же. Р. 143, 155]. Проблема, которая возникает при сопоставлении двух вариантов достижения воздержания от суждения, может быть решена путем гипотезы эволюции взглядов Секста от апоретизма энесидемовского типа к «классическому» варианту. Из 
должен прийти к выводу о непознаваемости вещей, то заявления о воздержании будут неискренни.

В данной работе мы намереваемся исследовать один из подобных аргументов, способных привести скептика к самоопровержению (по его рассмотрении будет ясна и возможность ответа на некоторые другие аргументы такого рода). Он касается тропа относительности и предполагает, что при его использовании Секст признает относительность всех вещей, из чего должно следовать принятие им релятивистских следствий, в том числе в виде утверждения о непостижимости природы вещей. Для этого мы сначала реконструируем троп относительности и заключенный в нем основной аргумент, затем кратко опишем «диалектическую стратегию» Секста и продемонстрируем, каким образом она позволяет ему отвести обвинение в негативном догматизме и тем самым в противоречии.

Г. Страйкер в своей статье о тропах Энесидема выделила две используемые в них стратегии: стратегию неразрешимости разногласий [16. Р. 121126] и стратегию относительности, которая в наиболее общем виде представлена в восьмом тропе [16. Р. 126-131]. Исследовательница подняла проблему, которая возникает при использовании тропа относительности, поскольку, как она сама отмечает, он принадлежит к негативно-догматической традиции [16. P. 133]. Если стратегия неразрешимости конфликтующих мнений создает искомое скептиком равновесие, то троп относительности показывает, что мы никогда не достигаем познания присущих вещи свойств по природе, что, как кажется, имеет следствием утверждение негативного догматизма. Страйкер считает поэтому, что троп относительности плохо вписывается в канон пирронизма Секста и признает, что не знает, как разрешить это затруднение, хотя очень кратко предлагает возможный путь решения [16. Р. 132].

Троп относительности (PH I 135-141) является ведущим тропом в ряду десяти тропов Энесидема (PH I 40-163), к нему восходят и все остальные (PH I 39). Он идет восьмым по счету и заметно отличается от остальных по содержанию ${ }^{1}$. Троп включает в себя ряд аргументов, и поэтому его можно считать целым аргументационным комплексом.

Рассуждение, представленное в нем, в общем виде можно реконструировать следующим образом, формально здесь используется стандартный modus ponens: если $p$, то $q$; $p$, следовательно, $q$. В заключение тропа проговаривается вывод: поскольку (1) «все вещи существуют по отношению к чему-нибудь», то (2) «ясно отсюда, что мы не можем сказать (le>gein ouj dunhso>meqa), какова каждая вещь по своей природе и в чистом виде (kata< th<n eJautou fu>sin kai< eijlikronw v), но только каковой она нам кажется по отношению к

гипотезы следует, что трактат $M$ является более ранним, чем $Р Н$. Другой вариант решения предложил Д. Мачука [13. Р. 147-150], показывая, что Секст использовал эти аргументы в своей диалектической игре с догматиками, не принимая вывод о несуществовании добра и зла по природе как истинный.

${ }^{1}$ Важным для понимания истории пирронизма является вопрос о характере тропа относительности в ряду десяти тропов, а также связь его с тропами Агриппы. Р. Бетт и П. Вудраф согласны в том, что Энесидем был апоретиком, т. е. опровергал позиции догматиков (в частности, стоиков), и что Секст приспособил аргументы под себя. (См. [14; 15. Р. 207-213]). Страйкер также замечает отличия тропа относительности от остальных в характере аргументации, и считает это свидетельством того, что он был изменен и приспособлен так, чтобы совмещаться с более абстрактными тропами Агриппы [16. P. 133]. 
чему-нибудь» (PH I 140). Секст по большей части (не только в этом тропе, но и в прочих из десяти) обосновывает первую посылку, приводя разные доводы и свидетельства в поддержку тезиса об относительности всех вещей и зависимости человеческого восприятия от состояния людей в конкретный момент времени и определенных условий. В этом тропе он явно выделяет двоякий смысл относительности: по отношению к судящему о вещи и отношение между наблюдаемыми вместе вещами (PH I 135). Для обоснования тезиса об относительности используется также ссылка на другие тропы Энесидема, в которых говорится о разнице в восприятиях в зависимости от телесных особенностей и предпочтений наблюдателя, от ситуаций, положений, расстояний, мест, условий и т. д.

Кроме того, Секст снабжает свое изложение восьмого тропа рядом абстрактных аргументов, «особым способом» обоснования относительности вещей (PH I 137-140). В них говорится о том, что любой предикат и всякая вещь так или иначе попадают в ситуацию относительности, поскольку к ним может быть применена категория сходства и инаковости, также все вещи состоят в родо-видовых отношениях, в отношениях знака и обозначаемого. И даже если мы признаем абсолютным (т.е. безусловно истинным) сам тезис о всеобщей относительности, он все равно сам будет относителен к нам как судящим. Иными словами, любой рассматриваемый предмет уже находится в отношении к рассматривающему его, и таким образом выпадает из разряда абсолютных (по природе) вещей ${ }^{1}$. Секст переформулирует этот аргумент в другом месте, когда говорит о тропе относительности в ряду пяти тропов Агриппы (PH I 177). Там говорится об относительности мыслимого и мыслящего его, и такое отношение обнимает вообще всякое познание в его классическом субъект-объектном понимании.

Здесь необходимо сделать отступление и вывести на свет скрытую посылку, лежащую в основании рассуждений в десяти тропах, которая делает осмысленным аргумент. С первого взгляда становится понятно, что Секст противопоставляет явления как доступное нам и потому бесспорное и очевидное, следовательно, не подлежащее исследованию (в PH I 22 он именует иx ajzh>thtov), скрытой природе вещей, которая как раз является предметом исследования ввиду своей неочевидности. Через все свои рассуждения (как минимум в десяти тропах) Секст подводит нас к мысли о том, что мы можем сказать о вещи в некотором ее отношении к чему-либо еще, о способе ее явленности нам, но при этом мы не познаем вещей по природе. Он часто пишет об этом в рассуждениях о тропах и заключает почти каждый из них словами о том, что различные ситуации, отношения и обстоятельства препятствуют постижению вещей как они есть по природе (pro $>\mathrm{v}$ th $<\mathrm{n} f \mathrm{u}>\sin$ ), или же говорит о воздержании от суждения о природе внележащих вещей (PH I 59, 78, 87, 93, $117,125,128,129,132,134,135,140,163)$. Можно сказать, что природа вещи понимается Секстом как существующая обособленно и абсолютно от всего иного, а значит, природа вещей не включает в себя относительные характеристики, которые доступны нам. Из этого следует, что любое отношение и от-

1 Несомненно, что «особые» аргументы вызывают по меньшей мере странное впечатление. Дж. Барнс и Дж. Эннэс называют их софистическими [17. Р. 140], а Г. Страйкер $\square$ эристическими [16. P. 126]. 
носительное свойство уже уводит нас от познания вещей по природе. Секст показывает, что все нам доступное может быть только относительным, поскольку как минимум самим фактом мышления вещи предстают нам относительными, но не такими, как они существуют по природе. Задача его состоит как раз в том, чтобы показать разными способами относительность всех восприятий вещей (перцептуальных и интеллектуальных), что он выполняет в десяти тропах.

Эта мысль наиболее отчетливо иллюстрируется следующими пассажами. В рассуждении к шестому тропу Секст пишет, что «если из подлежащих предметов ни один не воспринимается нами обособленно, но вместе с какимнибудь другим, то можно, пожалуй, сказать, каковой является смесь из внележащего предмета и того, вместе с чем она рассматривается, но нам нельзя было бы сказать, каковым является в чистом виде внележащий предмет» (PH I 124), и далее: «В силу примесей наши чувства не воспринимают точной сущности внешних предметов» (PH I 127). В седьмом тропе Секст пишет, говоря о внешних предметах: «Мы можем разбирать только соотношение их с чем-нибудь, но никоим образом не природу вещей самое по себе вследствие разноречивости представлений, происходящей от многосложности» (PH I 132). Но поскольку вещи никогда не воспринимаются не обособленно, их природа остается сокрытой от нас.

Итак, как минимум на основании относительности вещей, их доступные нам характеристики и свойства не могут считаться принадлежащими вещам, как они есть по природе ${ }^{1}$. Г. Страйкер пишет, что из посылки об относительности всех вещей и из посылки о том, что относительное не существует по природе, «следует, как кажется, что ничто не существует абсолютно, т.е. не существует природы вещей в смысле способа их существования самих по себе... Если мы сохраняем вторую посылку в неизменности, ведь необходимым кажется переход от «относительно» к «не по природе», мы приходим к заключению, что ничто не является существующим абсолютно». Далее она продолжает: «Это может быть истолковано так, что, насколько видит скептик, никакое свойство, по-видимому, не принадлежит никакой вещи по природе. И хотя в действительности может существовать некоторая природа вещей, мы не можем выяснить, какова она, и, следовательно, должны воздержаться от суждения об этом» [16. Р. 126].

Хотя Страйкер и отметила негативно-догматические свойства тропа относительности, неясным осталось, почему и как рассуждение приходит к необходимости воздержания от суждения, а не к отрицанию природы вещей. Страйкер вслед за Секстом не останавливается на недоступности абсолютных характеристик вещи и приходит к выводу о воздержании. Кажется очевидным, что, когда относительное исключается из ряда свойств природы вещей и все вещи попадают в класс относительных, Секст должен необходимо заключить к принципиальной непознаваемости природы вещей (или о ее отсутствии, что также неприемлемо для скептика в свете воздержания от суждения),

1 Такая интерпретация природы вещей воспринята Секстом, по всей вероятности, у Платона и догматиков платонического направления (восходящая к «пути сущего»у Парменида). Подлинно сущее должно быть неизменным, вечным, неподвижным и себе тождественным. Как будет показано, заимствование концепций оппонентов является важным приемом диалектической стратегии. 
поскольку с учетом сказанного представляется невозможным найти некоторую не-относительную, абсолютную характеристику. Однако он не делает этого, и вновь, как и в других тропах, констатирует воздержание от суждения. В самом начале восьмого тропа Секст представляет несколько иную версию вывода, нежели мы видели, но по той же схеме: (1) если «все существует по отношению к чему-нибудь», (2) то «мы удержимся говорить каково оно обособленное и по своей природе» (PH I 135). Как мы видим, посылка пропущена и остается невыясненным, каким образом может здесь наступить воздержание от суждения. В заключении к тропу перед нами предстает полный вывод: поскольку (1) «все вещи существуют по отношению к чему-нибудь», то (2) «ясно отсюда, что мы не можем сказать, какова каждая вещь по своей природе и в чистом виде, но только каковой она нам кажется по отношению к чему-нибудь». И уже отсюда следует (3), «что нам должно воздержаться от суждения о природе вещей» (РН I 140).

Из этих высказываний неясно, каким образом и на каком основании Секст не пришел к позиции негативного догматизма, поскольку своими аргументами он фактически исключил самую возможность познания неотносительных, несмешанных, а, значит и абсолютных вещей. Естественно было бы заключить к непостижимости вещей и тем самым принять сторону негативного догматизма. Здесь напрашивается аналогия, с одной стороны, с киренаиками, а с другой, более очевидная, - с релятивистами, ввиду использования тропа относительности ${ }^{1}$. Подобные учения, рефлексируя над относительностью или доступностью явлений, сокрытостью вещей по природе, приходят к негативному догматизму, хотя и различными путями ${ }^{2}$. Так или иначе, кажется, что и из тропа относительности следует (должен следовать) вывод о непознаваемости вещей по природе. Мы уже отмечали, какие следствия имеет это возражение для философии Секста.

Представляется, что Секст Эмпирик был достаточно осведомлен о последствиях использования аргументов и разработал определенные способы защиты. Сейчас рассмотрим возможные аргументы Секста, которые способны обеспечить ему защиту от подобных возражений и тем самым подробно

\footnotetext{
${ }^{1}$ Релятивизм Протагора отрицается в [18. Р. 166-169], где он доходит даже до отрицания ее наличия в Античности (18. Р. 168). Другие же, как, напр., К. Фогт [19. Р. 102-110], приписывают ее Протагору. Трудность заключается в том, что наиболее полным источником является платоновский «Теэтет», в котором под именем Протагора выведен образ закоренелого релятивиста. До сих пор актуален вопрос об исторической достоверности и сходстве персонажа диалога с реальным философом. Так или иначе, в Античности релятивизм присутствовал уже в виде умозрительной мысленной конструкции, берущей начало у Платона, даже если ни один философ в действительности не разделял его. Релятивистские аргументы принимались всерьез. Секст указывает, что Протагор впервые ввел троп относительности (PH I 216), но не конкретизирует его, так что трудно понять, о каком конкретно аргументе идет речь. С точки зрения концепции проблемного подхода становится ясно, что троп относительности Секста и обозначаемый им же таким наименованием аргумент Протагора нельзя необдуманно и поспешно отождествлять, поскольку мыслителей разделяет полтысячи лет; это означает, что сам характер ведущихся ими дискуссий был иным. По вопросу эпистемологической ориентации Протагора и последовавших возражений см. [20].

${ }^{2}$ Киренская школа учила о том, что нам доступны только аффекты, pa>qov, происходящие от вещей, но никогда не вещи по своей природе (Об отличии понимания ра>qov у киренаиков и Секста см. [21]). Таким образом, они объявляли вещи по природе непостижимыми (PH I 215). Кроме того, Секст дает некоторые основания для отнесения академиков к негативным догматикам, в частности Аркесилая и Карнеада (см. РН I 226, а также PH I 1-3). В то же время некоторые современные исследователи отвергают такую позицию [22].
} 
выяснить, как функционирует его исследование и, соответственно, как он может достичь атараксии. Тем самым мы выявим скрытые посылки, направляющие его исследования и аргументацию, и раскроем их статус в учении пирронизма. При этом окажется, что Секст пользовался особой пропозициональной установкой, которая определяла его отношение к исследованию и его правилам.

Среди современных специалистов по пирронизму при работе со скептическими аргументами популярностью пользуется концепция «диалектической стратегии». Разработку ее приписывают академическим скептикам с целью защиты от догматических аргументов в самопротиворечии и самоопровержении. Она восходит к диалогической практике Сократа (см. [24]), его вопросно-ответному способу беседы и его методу эленхуса, и была подхвачена Аркесилаем и использовалась академиками для критики стоиков. В целом же диалектический способ исследования был широко распространенным в философской практике Античности, а потому, очевидно, имел большие аргументативные ресурсы ${ }^{2}$.

Несомненно, что и Секст Эмпирик, наследуя скептической традиции и заимствуя аргументы академиков, знал и использовал этот метод в своей исследовательской практике. Здесь мы остановимся лишь на одном ее аспекте. В общем виде диалектическая стратегия заключается в (1) гипотетическом принятии посылок оппонента в ходе рассуждения, (2) для выявления внутренних противоречий и/или противоречащих следствий, для опровержения положений оппонента. Конечной целью этой практики являлось, конечно, устранение ложных мнений на пути поиска истины (см. [25]).

Секст Эмпирик, следуя первому шагу стратегии, разработал особый способ непропозициональной речи, характеризующий его сочинения и позволяющий ему не принимать истинность посылок по рассматриваемым вопросам. Примечательно то, что в РН Секст упоминает свой способ речи впервые именно в изложении тропа относительности (PH I 135; cp. также PH I 198, $M$ XI 18-20). Он разъясняет особенности скептического словоупотребления глагола «быть» (еi+nai). Используя этот глагол в речи в предложениях типа «x есть (e]sti) $y »$, где он выступает в качестве грамматической связки субъекта и предиката ${ }^{3}$, Секст каждый раз подразумевает под ним слово «является» или «мне кажется» (fai>nomai), и тогда предложение принимает форму для говорящего «x кажется мне/является мне $y »$. Иными словами, этот особый тип речи избегает всякого высказывания, будь то утверждение или отрицание, поскольку оно подразумевает наличие мнения (истинно/ложно, что $p$ ). К. Фогт называет это «правилом phainestai», согласно которому скептик сообщает лишь о своих переживаниях, pa>qov, не считая их содержание истинным или ложным ${ }^{4}$. Эта точка зрения находит подтверждение в разделе трак-

\footnotetext{
${ }^{1}$ Впервые эту стратегию на материале академиков выделил П. Кассен [23].

${ }^{2}$ Как минимум в платонической традиции. У стоиков диалектика совпадала с логикой в широком смысле (DL VII 41, 43). Диалектика как вопросно-ответный метод беседы для выявления истинных посылок среди общепринятых мнений разрабатывалась и Аристотелем в «Топике» и «Софистических опровержениях».

${ }^{3}$ Секст использует в своей речи копулятивное, а не экзистенциальное значение глагола «быть», cм. PH II 4, 10.

${ }^{4}$ O скептической речи см. [26. S. 72-79], подробно же см. до S. 128, а также [27].
} 
тата $P H$ «О невысказывании», где под «невысказыванием» (ajfasi>a) понимается такое состояние скептиков, когда они не выносят суждений о природе вещей, но лишь сообщают о своих впечатлениях по исследуемому вопросу. Секст отмечает, что он понимает невысказывание не в том смысле, что сама природа вещей такова, что не позволяет скептику выносить суждения о ней, но только сообщает о своем ментальном состоянии относительно исследуемого вопроса ввиду невозможности обоснованно предпочесть некоторый аргумент иному (РН I 192-193).

Отдельной и особой частью стратегии являются также рассуждения Секста в защиту способности скептика мыслить и вести исследования. Этой проблеме посвящено достаточное количество работ, поэтому мы не будем останавливаться на ней. Отметим лишь, что Секст указывает на очевидную способность понимать речь, которая имеется у всех людей, на наличие общего опыта и понятийного аппарата, с помощью которых скептик может понимать догматиков и их правила, с помощью которых он выстраивает критику их концепций, и противопоставлять им свои рассуждения, выстраивая их по правилам догматиков ${ }^{1}$. С учетом такой предпосылки скептик может рассуждать в рамках терминологии и правил тех, кто считает их истинными, и проверять концепции, правила и их применение на их соответствие собственным нормам.

Итак, в свете нашей проблемы это означает, что, когда Секст говорит «все вещи существуют относительно», под этим следует понимать выражение «все вещи кажутся [Сексту] относительными». Из этого ясно, что все высказывания его на этот счет не должны рассматриваться с точки зрения истинностного значения. Он ничего не говорит о том, каковы вещи по природе, придерживаясь невысказывания и выставляя лишь правдоподобный аргумент против равного ему по убедительности догматического. При этом важно помнить, что в диалектическом исследовании философ заимствует посылки, понятия и правила вывода у утверждающих нечто оппонентов. Это означает, что скептик также использует и их правила вывода, нормативные посылки, определяющие должное познание вещей по природе. Поэтому выводы, делаемые скептиками, не имеют для них обязательной силы и рассматриваются лишь как вероятные/убедительные, тогда как догматики, наоборот, обязаны принять их, поскольку выводы сделаны по их собственным правилам и исходя из их собственных посылок, которые они полагают истинными.

Таким образом, мы видим основания, стоящие за исследованием и позволяющие скептику избежать самопротиворечия - если он ничего не высказывает в строгом смысле слова, то не может возникнуть противоречия ввиду воздержания от суждения и, соответственно, самоопровержения. В то же время осуществляется диалектическая критика догматических позиций на основании их посылок и норм. К. Фогт, подчеркивая сократическую компоненту в диалектической практике пирронизма, полагает, что именно она спасает скептицизм от релятивизма [19. Р. 189].

${ }^{1}$ Об отношении скептика к нормам рационального исследования на примере закона противоречия см. [28]. О способности скептика мыслить см. [19. Р. 140-157; 29]. 
Однако нерешенными остаются некоторые проблемы, прямо следующие из применения диалектической стратегии Секстом Эмпириком. Чтобы показать, каким образом достигается воздержание от суждения, нужно выявить особенности использования диалектической стратегии у Секста, поскольку в своем классическом варианте (Сократ и академики) она является опровергающей. При этом нужно помнить, что воздержание от суждения происходит ввиду наличия двух paвных по убедительности противоречащих мнений. Данный вопрос мы рассмотрим в следующей работе.

\section{Литература}

1. Юм Д. Исследование о человеческом познании // Сочинения в двух томах. Т. 2. М.: Мысль, 1996.

2. Vogt K. Scepticism and action // The Cambridge Companion to Ancient scepticism / Ed. by R. Bett. Cambridge: Cambridge University Press, 2010. P. 165-180.

3. Bailey A. Pyrrhonian skepticism and the self-refutation argument // The philosophical quarterly. 1990. Vol. 40, No 158. P. $27-44$.

4. Kenny A. The Philosopher's History and the History of Philosophy // Analytic Philosophy and the History of Philosophy / Ed. by T. Sorell and G. Rogers. New York: Oxford University Press, 2005. P. 13-24.

5. Palmer J. Skeptical Investigation // Ancient Philosophy 20, 2000. P. 351-375.

6. Striker G. Sceptical Strategies // Doubt and dogmatism. Studies in Hellenistic philosophy / Ed. by M. Schoefield. Oxford: Clarendon Press, 1980. P. 54-83.

7. Burnyeat M. Protagoras and the self-refutation in Later Greek Philosophy // The Philosophical Review. 1976. Vol. 85, No 1. P. 44-69.

8. Striker G. Scepticism as a kind of Philosophy // Archiv für Geschichte der Philosophie. 2001. Vol. 83. Issue 2. P. 113-129.

9. Секст Эмпирик. Сочинения: в 2 т. / пер. А.Ф. Лосева, Н.В. Брюлловой-Шаскольской. М.: Мысль, 1976.

10. Sextus Empiricus. Pyrrhoniae Hypotyposes // Sextus Empiricus, Opera, vol. 1 / Ed. H. Mutschmann. Leipzig: Teubner, 1912. P. 3-209.

11. Диоген Лаэртский. О жизни, учениях и изречениях знаменитых философов / пер. М.А. Гаспаров; ред. А.Ф. Лосев. М.: Мысль, 1986. 571 с.

12. Bett R. Sextus's Against the Ethicists: Scepticism, Relativism or both? // Apeiron: A Journal for Ancient Philosophy and Science. 1994. Vol. 27, No. 2. P. 123-161.

13. Machuca D. Moderate Ethical Realism in Sextus' Against the Ethicists // New Essays on Ancient Pyrrhonism / Ed. By D. Machuca. Leiden: Brill, 2011. P. 143-178.

14. Woodruff P. Aporetic Pyrrhonism // Oxford Studies in Ancient Philosophy. Vol. VI. Oxford: Oxford University Press, 1988. P. 139-168.

15. Bett R. Pyrrho, his Antecedents, and his Legacy. Oxford: Oxford University Press, 2000. $264 \mathrm{p}$.

16. Striker G. The Ten Tropes of Aenesidemus // Essays on Hellenistic epistemology and ethics. Cambridge: Cambridge University Press, 1996. P. 116-134.

17. Barnes J., Annas J. The Modes of Scepticism. Ancient Texts and Modern Interpretations. New-York: Cambridge University Press, 1985. 204 p.

18. Bett R. The Sophists and Relativism // Phronesis. 1989. Vol. 34, No. 2. P. 139-169.

19. Vogt K. Belief and Truth. A Sceptic Reading of Plato. New-York: Oxford University Press, 2012. $209 \mathrm{p}$.

20. Lee M.-K. Epistemology after Protagoras. Responses to Relativism in Plato, Aristotle and Democritus. Oxford: Clarendon Press, 2005. 351 p.

21. O'Keefe T. The Cyrenaics vs. the Pyrrhonists on Knowledge of Appearances // New Essays on Ancient Pyrrhonism. Ed. By D. Machuca. Leiden: Brill, 2011. P. 27-40

22. Thorsrud H. Arcesilaus and Carneades // The Cambridge Companion to Ancient scepticism / Ed. by R. Bett. Cambridge: Cambridge University Press, 2010. P. 58-80.

23. Couissin P. Le stoicisme de la nouvelle Academie // Revue d'historie de la philosophie. 1929. 3. P. 241-276. 
24. Vlastos $G$. The Socratic Elenchus // Oxford Studies in Ancient Philosophy. Oxford: Oxford University Press, 1983. Vol. I. P. 37-58.

25. Vogt K. The Aims of Skeptical Investigation // Pyrrhonism in Ancient, Modern and Contemporary Philosophy / Ed. by D. Machuca. New York: Springer, 2011. P. 33-50.

26. Vogt K. Skepsis und Lebenspraxis. Das pyrrhonische Leben ohne Meinungen. München: Verlag Karl Albert, 1998. 202 S.

27. La Sala $R$. Die Züge des Skeptikers. Der dialektische Charakter von Sextus Empiricus` Werk. Göttingen: Vandenhoeck und Ruprecht, 2005. 204 S.

28. Machuca D. Pyrrhonism and the Law of Non-Contradiction // Pyrrhonism in Ancient, Modern and Contemporary Philosophy / Ed. by D. Machuca. New York: Springer, 2011. P. 51-77.

29. Маслов Д.К. Скептическая способность мыслить // Вестник НГУ. Серия «Философия». 2015. Т. 3. С. $157-163$.

Maslov Denis K. Institute of Philosophy and Law, SB RAS (Novosibirsk, Russian Federation).

E-mail: denn.maslov@gmail.com

DOI: $10.17223 / 1998863 X / 37 / 8$

THE RELATIVITY MODE AND «DIALECTICAL STRATEGY» IN SEXTUS EMPIRICUS.

Key words: the Relativity Mode, dialectical strategy, Sextus Empiricus, Ancient Pyrrhonism, Self-Refutation.

The goal of Sextus Empiricus' philosophy implies achieving the tranquility of soul (ataraxia), which is reached through investigation of scientific and philosophical beliefs. Sceptic comes to conclusion, Sextus says, that conflicting beliefs have equal persuasiveness, which allows him to suspend judgement about truth and falsity of any issues under investigation.

At the same time Sextus uses powerful arguments of negative dogmatic nature, what provides reasons to some scholars to contend Sextus' insincerity and inconsistency. Indeed, we come to strange results, undertaking analysis of the Relativity Mode. If we reconstruct arguments, contained in the Mode, we can see that the main premise of the Mode is an assumption that relative knowledge of the things is not the proper one, for it cannot reveal their real nature. For the being of things in themselves is assumed as absolute and not relative one. Every relative predicate then does not reveal the real properties of the things in themselves. Assuming this, one should certain conclude that things are incomprehensible, which means in its turn taking agnosticism. However this takes no place in Sextus' case at the end of every Mode he declares necessity of suspending judgement.

This issue raises the main problem of this paper. I argue that Sextus has sufficient means to refute those hasty charges sketched above. So it is proposed an apologetic interpretation based on so called «dialectical strategy», which can be traced to Socrates and which was inherited by Sextus through Arcesilaus. It consists in hypothetical assuming premises and deduction rules of opponents (dogmaticists), and revealing internal contradictions of their accounts. As example of such strategy could be taken a special way of skeptical speaking, which is described by «phainestai rule» (K. Vogt). According to this rule the pyrrhonian sceptic makes no statements, but just utters about his perceptions and mental states. It means that the sceptic have a special skeptical propositional attitude, according to which he makes no truth claims but he is just reasoning from the dogmatist's point of view. Thus we reach a point that the sceptic doesn't argue truth of premises and deduction rules, and thereby is not committed to their conclusions. This shows why the sceptic isn't entitled to claim agnosticism as consequence of his reasoning.

However this interpretation calls an account of isosthenia in question, which I'll undertake in the following paper.

\section{References}

1. Hume, D. (1996) Sochineniya v dvukh tomakh [Works in two vols]. Vol. 2. Moscow: Mysl'.

2. Vogt, K. (2010) Scepticism and action. In: Bett, R. (ed.) The Cambridge Companion to Ancient skepticism. Cambridge: Cambridge University Press. pp. 165-180.

3. Bailey, A. (1990) Pyrrhonian skepticism and the self-refutation argument. The Philosophical Quarterly. 40(158). pp. 27-44. DOI: 10.2307/2219965 
4. Kenny, A. (2005) The Philosopher's History and the History of Philosophy. In: Sorell, T. \& Rogers, G. (eds) Analytic Philosophy and the History of Philosophy. New York: Oxford University Press. pp. 13-24.

5. Palmer, J. (2000) Skeptical Investigation. Ancient Philosophy. 20. pp. 351-375.

6. Striker, G. (1980) Sceptical Strategies. In: Schoefield, M. (ed.) Doubt and dogmatism. Studies in Hellenistic philosophy. Oxford: Clarendon Press. pp. 54-83.

7. Burnyeat, M. (1976) Protagoras and the self-refutation in Later Greek Philosophy. The Philosophical Review. 85(1). pp. 44-69. DOI: 10.2307/2184254

8. Striker, G. (2001) Scepticism as a kind of Philosophy. Archiv für Geschichte der Philosophie. 83(2). pp. 113-129. DOI: 10.1515/agph.83.2.113

9. Sextus the Empiricist. (1976) Sochineniya: $v 2 t$. [Works. In 2 vols]. Translated by A.F. Losev, N.V. Bryullova-Shaskolskaya. Moscow: Mysl'.

10. Sextus the Empiricist. (1912) Opera. Vol. 1. Leipzig: Teubner. pp. 3-209.

11. Diogenes of Laertius. (1986) O zhizni, ucheniyakh i izrecheniyakh znamenitykh filosofov [On the life, teachings and sayings of the famous philosophers]. Translated by M.A. Gasparov. Moscow: Mysl'.

12. Bett, R. (1994) Sextus's Against the Ethicists: Scepticism, Relativism or both? Apeiron: A Journal for Ancient Philosophy and Science. 27(2). pp. 123-161. DOI: 10.1515/APEIRON.1994.27.2.123

13. Machuca, D. (2011) Moderate Ethical Realism in Sextus' Against the Ethicists. In: Machuca, D. (ed.) New Essays on Ancient Pyrrhonism. Leiden: Brill. pp. 143-178.

14. Woodruff, P. (1988) Aporetic Pyrrhonism. In: Annas, J. (ed.) Oxford Studies in Ancient Philosophy. Vol. VI. Oxford: Clarendon Press. pp. 139-168.

15. Bett, R. (2000) Pyrrho, his Antecedents, and his Legacy. Oxford: Oxford University Press.

16. Striker, G. (1996) Essays on Hellenistic epistemology and ethics. Cambridge: Cambridge University Press. pp. 116-134.

17. Barnes, J. \& Annas, J. (1985) The Modes of Scepticism. Ancient Texts and Modern Interpretations. New-York: Cambridge University Press.

18. Bett, R. (1989) The Sophists and Relativism. Phronesis. 34(2). pp. 139-169. DOI: $10.1163 / 156852889 X 00107$

19. Vogt, K. (2012) Belief and Truth. A Sceptic Reading of Plato. New-York: Oxford University Press.

20. Lee, M.-K. (2005) Epistemology after Protagoras. Responses to Relativism in Plato, Aristotle and Democritus. Oxford: Clarendon Press.

21. O'Keefe, T. (2011) The Cyrenaics vs. the Pyrrhonists on Knowledge of Appearances. In: Machuca, D. (ed.) New Essays on Ancient Pyrrhonism. Leiden: Brill. pp. 27-40

22. Thorsrud, H. (2010) Arcesilaus and Carneades. In: Bett, R. (ed.) The Cambridge Companion to Ancient skepticism. Cambridge: Cambridge University Press. pp. 58-80.

23. Couissin, P. (1929) Le stoicisme de la nouvelle Academie. Revue d historie de la philosophie. 3. pp. 241-276.

24. Vlastos, G. (1983) The Socratic Elenchus. In: Annas. J. (ed.) Oxford Studies in Ancient Philosophy. Vol. I. Oxford: Clarendon Press. pp. 37-58.

25. Vogt, K. (2011) The Aims of Skeptical Investigation. In: Machuca, D. (ed.) Pyrrhonism in Ancient, Modern and Contemporary Philosophy. New York: Springer. pp. 33-50.

26. Vogt, K. (1998) Skepsis und Lebenspraxis. Das pyrrhonische Leben ohne Meinungen [Skepticism and lifestyles. The Pyrrhonic life without opinions]. München: Verlag Karl Albert.

27. La Sala, R. (2005) Die Züge des Skeptikers. Der dialektische Charakter von Sextus Empiricus' Werk [The Traits of the Skeptic. The dialectical character of Sextus Empiricus' work]. Göttingen: Vandenhoeck und Ruprecht.

28. Machuca, D. (2011) Pyrrhonism and the Law of Non-Contradiction. In: Machuca, D. (ed.) Pyrrhonism in Ancient, Modern and Contemporary Philosophy. New York: Springer. pp. 51-77.

29. Maslov, D.K. (2015) Skepticheskaya sposobnost' myslit' [Skeptical ability to think]. Vestnik NGU. Seriya “Filosofiya”. 3. pp. 157-163. 